\title{
Linx
}

Revue des linguistes de l'université Paris X Nanterre

$8 \mid 1996$

Du dire et du discours

\section{Le mot autonomie dans les projets éducatifs des municipalités : tentative de définition}

\section{Mustapha Krazem}

\section{OpenEdition}

\section{Journals}

Édition électronique

URL : http://journals.openedition.org/linx/1151

DOI : 10.4000/linx.1151

ISSN : 2118-9692

\section{Éditeur}

Presses universitaires de Paris Nanterre

\section{Édition imprimée}

Date de publication : 1 septembre 1996

Pagination : $91-110$

ISSN : 0246-8743

\section{Référence électronique}

Mustapha Krazem, «Le mot autonomie dans les projets éducatifs des municipalités : tentative de définition », Linx [En ligne], 8| 1996, mis en ligne le 13 juillet 2012, consulté le 19 avril 2019. URL: http://journals.openedition.org/linx/1151 ; DOI : 10.4000/linx.1151

Ce document a été généré automatiquement le 19 avril 2019

Département de Sciences du langage, Université Paris Ouest 


\title{
Le mot autonomie dans les projets éducatifs des municipalités : tentative de définition
}

\author{
Mustapha Krazem
}

1 Comme n'importe quelle activité intellectuelle, l'éducation possède un vocabulaire spécifique. Il se caractérise par la faible exploitation des possibilités néologiques offertes par la langue. Il privilégie l'appropriation de termes et leur attribue une signification particulière. Il semble cependant qu'à la différence d'autres domaines intellectuels, le sens des expressions techniques ainsi réinvesties est, au moins à première vue, plus fragile, plus malléable. L'objectif premier de notre étude est de prendre la mesure de cette fragilité afin de cerner les points de vue qui la rendent perceptible. Pour atteindre ce but, le mot autonomie présente toutes les qualités requises. Il n'est pas propre à l'éducation mais l'éducation le dote de valeurs techniques, en témoignent les titres d'ouvrages théoriques récents (Vayer 1993 et Hofmanns-Gosset 1994), qui revendiquent pleinement leur appartenance au champ des sciences de l'éducation ${ }^{1}$. Ces ouvrages sont une preuve de l'emploi fréquent de ce terme par les doctrines éducatives. Mais l'autonomie a largement franchi la barrière théorique. Les praticiens, quelle que soit leur fonction (enseignant, éducateur, animateur...) l'ont adoptée. Aussi avons-nous souhaité en connaître l'opportunité dans l'articulation entre la «théorie » et la "pratique ». A cette fin, nous avons choisi d'étudier l'autonomie à travers les définitions qui émanent des projets éducatifs des communes parce que ces derniers sont le lieu de rencontre désiré. En effet, ces textes, écrits par des élus municipaux, s'adressent à des «techniciens » et reproduisent les fondements idéologiques des actions éducatives dépendant des communes.

2 Nous n'ignorons pas la nature politique indéniable du projet éducatif. Nous en profiterons pour tester la résistance de l'autonomie lorsqu'elle est confrontée aux enjeux idéologiques. Nous verrons qu'elle en sort renforcée. Mieux même, l'autonomie est unanimement appropriée sans discussion. Plus précisément, c'est le mot qui suscite la 
bienveillance générale, devenant de ce fait fédérateur. Evidemment, ce consensus s'expose à quelques suspicions, assurément fondées lorsqu'on s'attache à décortiquer le contenu définissant l'autonomie. Mais cela n'est pas primordial. En effet, au-delà des divergences de sens souvent inconciliables, au-delà des discours politiques, l'étude des projets renforce l'importance de l'énonciateur, de son discours, dans une théorie éducative. En gros, on distingue autant de définitions que d'énonciateurs.

\section{Présentation du corpus}

3 Le corpus que nous avons exploité est composé de textes peu connus: les projets éducatifs des communes. Le projet éducatif est un écrit réglementaire exigé par le ministère de la Jeunesse et des Sports depuis 1984 pour habiliter l'ouverture des centres de loisirs (mercredis, accueils pré ou post scolaires). Ce corpus nous a paru intéressant en raison des nombreux attraits qu'il recèle et que nous décrivons à présent.

4 Le secteur parascolaire prend en France une place grandissante. Depuis une bonne dizaine d'années il connaît un développement imposant, attesté par l'augmentation constante des crédits alloués. Différentes raisons y concourent. Les besoins (et les demandes) d'accueil d'enfants progressent régulièrement. Parallèlement, le parascolaire est reconnu comme une réponse d'urgence à certaines conséquences de la crise économique. On comprend pourquoi le loisir des enfants est devenu un argument électoral qui a connu un écho national lors de la campagne des municipales de Juin 95.

Le personnel d'encadrement a vécu lui aussi une mutation considérable. Ce secteur est devenu pourvoyeur d'emplois stabilisés. Les saisonniers, les occasionnels et à plus forte raison les quasi-bénévoles ont fortement diminué. Ils ont cédé la place à un autre type sociologique d'encadrement pour lequel le ministère a conçu des formations professionnelles. Certaines communes profitent d'ailleurs des concours administratifs pour effectuer leur recrutement. Du coup, les missions dont ce secteur est chargé ont augmenté. A l'origine simples garderies ou patronages, les centres de loisirs collaborent aujourd'hui avec l'école dans de nombreuses villes (ateliers dans la classe, aide aux devoirs...). On y voit fréquemment une solution d'avenir'2. Ce bouleversement n'est pas sans conséquences sur la teneur des projets éducatifs. L'idéologie de cet écrit prévoit sa propre application par les principaux destinataires qui sont des techniciens, "professionnels» pour certains. Le projet éducatif n'est donc pas un texte de vulgarisation dans lequel l'autonomie serait capturée dans des combinaisons inhérentes à la reformulation du discours scientifique.

6 Néanmoins, la raison première motivant notre choix n'est pas la justification sociale du corpus. A notre connaissance, le projet éducatif est le seul texte qui oblige des élus à formuler un discours sur l'éducation. De plus, l'intérêt de ce texte n'étant pas encore véritablement reconnu, les auteurs jouissent d'une liberté d'écriture qui n'est ni cadrée par les appareils politiques, ni modérée par les « usagers». Nous avons donc rassemblé trente et un projets éducatifs provenant de vingt-neuf communes, la plupart situées en région parisienne (27/29) sauf Dreux (Eure-et-Loir) et Nanteuil le Haudouin (Oise). La plus 
importante a quatre-vingt-dix mille habitants, la plus petite trois mille. En tout, plus d'un million d'habitants sont ou ont été touchés par ces projets.

Plusieurs variables nuisent à l'homogénéité du corpus, mais elles ne dénaturent pas selon nous les résultats obtenus :

- (i) La surreprésentation de l'lle de France ne doit pas surprendre. En effet, ce secteur éducatif est particulièrement développé en région parisienne du fait des missions nouvelles lui incombant, plus criantes en zone urbaine.

- (ii) La couleur politique des communes est variée sans toutefois que nous ayons cherché à respecter une représentativité réelle. Bien entendu, les quatre grandes familles politiques figurent dans notre étude. Nous indiquerons dans le tableau des résultats l'origine politique des projets au moment de leur rédaction puisque ceux-ci ont été rédigés entre 1984 et 1995.

- (iii) Nous avons inséré deux variations dans notre corpus. La ville de Massy est représentée deux fois : par le projet antérieur aux municipales de 95 et par son successeur, né du changement de majorité aux élections de Juin (U.D.F à la place de P.S.). De plus, nous avons intégré les deux projets que nous a fournis la ville de Bobigny (centres de loisirs et centres de vacances).

8 Le corpus est certes hétérogène mais cela n'handicape pas notre but. Notre étude ne se veut pas représentative. Elle tente simplement de pointer quelques faits que l'analyse du langage contribue à mettre en relief. C'est pourquoi nous ne livrerons pas de résultats sous forme de pourcentage car ils usurperaient la rigueur scientifique reconnue à la mesure.

9 Un dernier aspect du corpus mérite une halte explicative. Il s'avère que le ministère n'impose aucune contrainte méthodologique ou qualitative. Seul, le fait de produire de l'écrit est réclamé. D'ailleurs, la rédaction est le point essentiel nouveau de l'arrêté ministériel de 1984. En obligeant les communes à formuler leurs conceptions éducatives, le ministère espère tenir un gage de qualité supplémentaire. En conséquence, on trouve des projets très différents. Les plus courts $(6 / 31)$ ne font qu'une page, le plus long en a dix-huit (moyenne 4 pages et demie). Les propos idéologiques sont, on l'imagine sans peine, très variables. Tel projet entre dans des réflexions aussi précises que l'organisation du goûter des enfants (30-Le Vésinet) pendant que tel autre (13-Massy) appelle seulement son personnel à inscrire ses animations et ses choix d'organisation en conformité avec la convention internationale des droits de l'enfant, ce qui, au moins en première analyse, est le cadre minimum de toute action en direction de l'enfance ${ }^{3}$.

10 L'absence de contrainte a eu des incidences sur notre recherche. Faute d'une méthodologie officielle d'élaboration des projets éducatifs (ce qu'on peut comprendre), la structuration du texte ne permet pas toujours d'en récupérer l'organisation hiérarchique. Pour apprécier si un élément $\mathrm{x}$ relevait de la définition de l'autonomie, nous nous sommes heurté quelquefois à l'impossibilité de prendre une décision.

11 Il n'est pas garanti que ce soient les élus eux-mêmes qui aient rédigé le projet éducatif. L'analyse des traces d'énonciation nous a peu secouru. Peu de projets sont signés (4/31). D'autres se servent de «nous », parfois « on », ce qui suppose un énonciateur même non identifié. Nous signalons également des marques de métalangage sur le projet, ce qui dissocie l'énoncé de son énonciateur. On présume alors sa présence (1 et 2):

(1) Ce document s'enrichira de la réflexion des différents partenaires. (14-Gennevilliers) 
(2) Il est donc nécessaire d'établir un projet éducatif. (25-Montigny le Bretonneux)

les causes de cette pluralité impliquait dans un premier temps de mettre à plat les éléments constitutifs de l'autonomie avant de les déployer dans un dispositif à vocation comparative. Nous avons donc choisi, pour exposer les résultats, de construire un tableau (cf. p. 106). Mais auparavant, les différents items que nous avons estimés pertinents à l'examen du corpus seront commentés. Nous avons éliminé ceux qui n'apparaissaient qu'une seule fois. Les résultats sont consignés au moyen des marques +/-. Sauf indication contraire, le signe + inscrit dans une colonne signifie que le critère est constitutif de l'autonomie d'après la ville étudiée. Le signe - signale que le critère est consigné dans le projet mais qu'il dépend de la définition d'une autre expression ${ }^{4}$. La case vide peut exprimer deux faits : soit que le critère est purement et simplement absent, soit, cas plus rare, que sa présence ne peut être associée ou écartée d'une manière certaine.

ne autre donnée est nécessaire à la lecture du tableau. Rappelons-le, le ministère n'a aucune exigence sur la teneur des projets. La présence du mot autonomie n'est donc pas requise $^{5}$. Pourtant, on le récupère dans la plupart des projets (23/31), doté, nous le verrons, d'acceptions très différentes. Les huit autres projets ne sont pas pour autant bâclés. Eux aussi s'attachent à élaborer une idéologie destinée à leurs structures. Le vocabulaire employé (vie collective, responsabilité, épanouissement... ) ne les distingue pas. Il s'apparente également à un vocabulaire technique ${ }^{6}$. C'est pourquoi nous avons souhaité intégrer une partie du contenu de ces projets dans le tableau de résultats. Nous y avons fait figurer les éléments en lien avec notre recherche qui apparaissaient dans les autres projets. L'opposition +/- n'étant pas alors pertinente, nous avons décidé de recourir au signe + pour noter la présence de l'élément. Le projet (2-Courbevoie) par exemple parle de la liberté ce qui justifie une marque + pour cet élément. 


\section{Un mot positif}

16 L'autonomie est un mot positif. C'est ainsi que l'éducation le perçoit. Sur ce point, autonomie affiche une similitude immédiate avec liberté qui, ainsi que le montre Petiot (1990) est «un terme valorisant et valorisé... ce sur quoi se rencontrent tous les débatteurs ». Une différence majeure est cependant décelable. L’a priori positif de l'autonomie s'étend sans heurt au contenu imaginé. La formulation du signifiant autonomie ignore la fragilité de son signifié. Aussi sommes-nous amené à considérer ce terme comme fédérateur dans le milieu de l'éducation. L'autonomie y est brandie par tous, appréhendée dans un consensus qui n'a pas conscience de son universalité, aveugle à la couleur politique des majorités municipales. Les projets sont tous d'accord sur le mot qu'ils croient pourvu d'un contenu indubitable. Pourtant, que l'autonomie ait un goût naturellement positif ne va pas de soi. Hors du contexte de l'éducation, elle a été (et est encore) l'origine de désaccords et quelquefois de conflits armés. Etre pour l'«autonomie de Gaza et Jericho » implique qu'être contre est licite. Les avis sont partagés sur les «autonomistes» de l'E.T.A. Point de cela dans l'éducation: «être pour l'autonomie » n'est pas pertinent puisqu'il n'est pas envisageable « d'être contre ». ${ }^{7}$ Notre corpus décèle toutefois quelques failles dans cette unanimité.

17 Revenons d'abord sur les projets « dépourvus » d'autonomie. Ils sont huit. Que faire dire à ce silence? Rien d'absolu bien entendu. Mais nous n'écartons pas l'éventualité d'une omission volontaire surtout lorsque les projets sont consistants. Cette omission serait attribuable à une distanciation envers le terme lui-même et non envers un contenu supposé puisqu'on isole plusieurs points communs. Ce qui est inclus, dans la définition de l'autonomie, des projets qui s'y réfèrent est cité dans les projets qui ne s'en servent pas.

Ensuite, l'environnement linguistique du terme dévoile quelques cas, trois exactement, où les projets se méfient du contenu potentiel du concept. Les adjectifs accolés et certains $\mathrm{GN}$ introducteurs en témoignent. Nous trouvons ainsi : une certaine autonomie (10-Noisy le Sec et 22-St Michel/Orge), une certaine forme d'autonomie (14-Gennevilliers), la marge d'autonomie et de liberté (22-St Michel/Orge). Cette distance est relative et ne remet pas véritablement en cause l'aspect positif de l'autonomie, mais elle crée un nouvel objet qui n'apparaît pas ailleurs : une autre autonomie. De ce fait, on peut légitimement penser que selon ces projets le terme cesse d'être fédérateur en lui-même et appelle un contenu. Il n'en est rien. Contrairement à d'autres, ils ne définissent pas notre sujet d'intérêt (cf. p. 104). Ils n'éclaircissent pas ce que serait une "mauvaise» autonomie. Comment expliquer alors cette distanciation? Quelques hypothèses dont la justesse n'est pas assurée sont plausibles. D'abord, nous n'écartons pas le fait que l'autonomie étant positive a priori, en postuler une mauvaise serait susceptible de troubler l'interlocuteur habitué à la considérer comme une part substantielle de sa profession et de sa formation. Comment un journaliste recevrait-il «une certaine liberté d'expression»? Ensuite, soulignons que ces trois communes sont communistes ou d'union de la gauche. L'autonomie y est peut-être dotée d'une résonance particulière, non compatible avec certaines positions historiques d'une partie de la gauche. On pense ici aux «syndicats autonomes » ou bien encore à une appropriation politique plus récente du terme par une

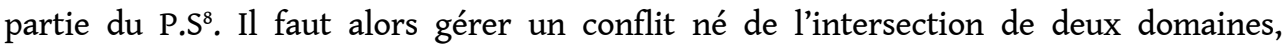
l'éducatif et le politique et accomoder des connotations opposées venant de deux champs qui se croisent peu'. Enfin, nous n'écartons pas l'hypothèse que l'autonomie soit entendue 
comme synonyme d'égoïsme ou d'individualisme. Ce point n'est pas confirmé par les textes de notre corpus, mais on le déniche dans Hoffmans-Gosset (1994), qui se fait le relais d'une opinion souvent répandue. L'auteur ressent clairement le besoin d'affirmer que « l'autonomie n'est pas l'individualisme ». Curieux besoin en vérité, sauf à considérer l'individu dans les limites que la société entend lui donner, par autonomie interposée. Nous y reviendrons.

\section{La place de l'autonomie dans la stratégie éducative}

19 Afin d'éclaircir la sémantique de l'autonomie, nous nous sommes intéressé aux propriétés présupposées par l'emploi du terme, antérieures à toute démarche consciente de l'énonciateur. Cette recherche est primordiale pour notre objectif. Dès ce préliminaire, nous nous heurtons à des divergences nettes voire inconciliables. En conséquence, nous avons classé tous les éléments entrant dans la configuration suivante :

(3) $\mathrm{N}_{\text {agent }} \mathrm{V}$ autonomie de $\mathrm{N}_{\text {objet }}$ si $\mathrm{N}_{\text {condition }}$

Chaque fragment de cette configuration nous renseignera. Nous débuterons par l'objet de l'autonomie, c'est à dire à qui ou à quoi elle s'applique. Puis, nous récupérerons la signification provenant du sémantisme de l'objet sélectionné par les verbes introducteurs, avant de nous intéresser aux agents. Enfin, nous recenserons quelques conditions nécessaires à la réalisation du concept.

\section{Posture de l'énonciateur}

21 Préalablement, il est nécessaire de dégager en amont la façon dont l'énonciateur considère la configuration (3). Ses manifestations peu nombreuses ne doivent pas masquer l'intérêt qu'il nous procure. Afin de rendre ce souci opératoire, nous avons jugé indispensable de compléter la configuration (3) par une marque explicite d'énonciation. Nous débusquerons par ce biais ce que nous tiendrons pour la fonction de l'autonomie.

(4) Enonciateur pense que $\left[\mathrm{N}_{\text {agent }} \mathrm{V}\right.$ autonomie de $\mathrm{N}_{\text {objet }}$ si $\left.\mathrm{N}_{\text {condition }}\right]$ est $\mathrm{N}_{\text {fonction }}$

Il s'avère en effet que la fonction de l'autonomie est l'aspect immédiatement quantifiable résultant d'une investigation liée à la subjectivité, à "l'appréciation » des énonciateurs (Beacco/Moirand 1995). Quelle est cette fonction? Comment est-elle considérée dans une action éducative? La question est cruciale puisque le ministère lui suppose des conséquences dans la traduction concrète des principes éducatifs des élus. On relève ici deux cas : l'autonomie est (i) un moyen ou (ii) une fin en soi. Ces deux possibilités s'excluent mutuellement ${ }^{10}$. On recherche (i) en sélectionnant certaines déterminations. D'après quelques projets, l'autonomie est un canal décisif dans l'accomplissement d'une fraction d'idéal. Idéal philosophique (5) ou plus matériel (6) : 
(5) Vers une autonomie au sein d'une vie collective qui offre à chacun la possibilité d'exercer sa citoyenneté. (8-Chatenay)

(6) [le personnel a] appris à cet enfant à devenir autonome à table de façon à ce qu'il ait acquis la dextérité souhaitée pour son passage dans les restaurants scolaires primaires. (25Montigny)

La valeur (ii) est, quant à elle, une valeur par défaut. Il semble aller de soi que l'autonomie constitue un but. Elle n'est donc jamais affectée à des fins strictement descriptives. La forme adjectivale est rarement attestée. Autonome est parfois épithète, mais quand c'est le cas, le nom qualifié se comporte pareillement à l'élément neutre d'une opération. Ainsi, dans le développement autonome ou l'individu autonome, c'est l'autonomie qui est renforcée. Le nom qualifié sert de support à l'autonomie comme les demi-points dans le comptage final d'une partie de tarot. La valeur (ii) est très fréquente dans les divers projets. A preuve, l'autonomie y est souvent coordonnée avec l'épanouissement, ce qui, au minimum, leur accorde un statut similaire. Or, nous semble-t-il, l'épanouissement est pour un individu un but et non pas un moyen. Sinon, (ii) est linguistiquement marquée lorsqu'elle est modélisée sous forme d'objectif $^{11}$, sans quoi on la cerne dans le discours sans distinction syntaxique particulière :

(7) Donner des moyens pour accéder à davantage d'autonomie. (1-St Cloud)

(8) La conception des salles de restaurants donne à l'enfant les moyens d'une autonomie qui s'apprend jour après jour. (31-Marly)

\section{L'objet de l'autonomie}

Le Robert électronique nous fournit trois classes de noms susceptibles de rentrer dans la formule (9), conçue pour rendre compte de l'objet de l'autonomie :

(9) N est autonome, l'autonomie de $\mathrm{N}$

26 (a) un individu, (b) un groupe d'individus ou (c) un objet vu principalement sous l'angle de la quantité d'énergie dont il dispose pour fonctionner.

Nous acceptons sans discussion que, bien que large et pas toujours aisé à définir, l'objet de l'éducation écarte (c). Mais, rien n'empêche (a) et (b). Curieusement, seul le sens lié à l'individu est répertorié dans notre corpus. L'autonomie de l'enfant est la formulation la plus banale. Nous ne repérons pas d'occurrences du type les groupes autonomes. Certes, nous rencontrons fréquemment la séquence l'autonomie des enfants. Cette formulation autorise les deux interprétations (a) et (b). Toutefois il n'y a jamais d'ambiguïté. Le projet 22 (St Michel) est particulièrement significatif à cet égard. Quand il décrit les actions les 
plus diverses en direction des enfants, il a recours au pluriel. En revanche, enfant est au singulier dès qu'il est associé à autonomie. Dans l'éducation, l'autonomie est bel et bien individuelle, ce que confirment ostensiblement Vayer (1993) et Hofmanns-Gosset (1994) ${ }^{12}$.

Cette apparente unité des projets ne fournit pas pour autant une base commune de définition véritablement décisive. Nous y voyons surtout le signe que l'autonomie a acquis un statut technique. Un argument supplémentaire le confirme: une recherche dans des textes plus anciens (réglementations, manuels pédagogiques, présentation de mouvements d'éducation populaire) établit qu'avant le début des années soixante, le mot autonomie n'est pas encore doté d'une valeur technique. Il est certes utilisé quelquefois, mais indifféremment avec les sens a et $b^{13}$.

Est-il possible de préciser davantage l'individu dont il est question? A peine. On le constate sans surprise, l'enfant est toujours l'objet de l'autonomie ${ }^{14}$ (colonne A4b). Mais on remarque un moins grand élan pour associer l'adulte à l'autonomie (colonne A4a). Cela n'est pas accessoire. Puisqu'elle est très fréquemment un but, l'autonomie implique une localisation dans le temps, que baliserait par exemple la référence à l'âge adulte.

\section{Statut de l'autonomie}

L'étude des verbes introducteurs révèle plusieurs éléments de sens. Un verbe sélectionne des noms ou plutôt sélectionne dans ce nom des sèmes compatibles. Il en va de même pour les verbes appelant l'autonomie, qu'ils aient conservé leur catégorie initiale ou qu'ils soient nominalisés. La liste que nous avons dressée souligne la diversité des acceptions choisies. Nous croyons discerner trois orientations principales :

(i) L'autonomie est une propriété de l'individu, innée, assimilable à un droit que l'on exerce ou non (colonne A3a). Dans cette optique, la quantification est secondaire : on est ou on n'est pas autonome ou plutôt on a le droit ou on n'a pas le droit de l'être. Des énoncés du type le droit à, permettre, autoriser l'autonomie induisent (i). Nous relevons encore d'autres introducteurs comparables : garantir, respecter, être placé en position de. Dans le corpus, nous n'observons que très peu d'occurrences de cette espèce. Elle n'apparait jamais seule (sauf 28-Bobigny) ${ }^{15}$.

(ii) L'autonomie est une qualité présente chez l'enfant (plutôt que chez l'individu) qu'il faut encourager. La quantification est ici nécessaire aux actions envisagées. L'enfant a de l'autonomie mais il faut qu'il en ait davantage : l'autonomie est alors un parcours ${ }^{16}$. Le sens (ii) s'incarne dans la séquence développer l'autonomie, maintes fois employée. Dans quelques cas, on découvre des empreintes linguistiques de la quantification: plus, davantage d'autonomie, qui s'apprend jour après jour (31-Marly). Néanmoins, elles ne sont pas spécifiques de (ii). Elles sont compatibles avec le type suivant.

(iii) L'autonomie est une (ou des) aptitudes ignorées de l'enfant. Le crucial, c'est l'absence initiale de l'autonomie. La théorie éducative souhaite que l'enfant l'acquière. Nous le devinons à travers des énoncés du type apprendre, accéder à, acquérir, parvenir à l'autonomie. La quantification est moins essentielle au contenu transitif de ces verbes. Le sème décisif est la concrétisation du résultat. L'autonomie devient évaluable, le plus souvent parce qu'elle se démultiplie en objectifs plus précis. La traduction pratique s'en trouve facilitée mais on doute alors de l'intérêt de convoquer le mot autonomie puisque celui-ci se redéploie sur d'autres expressions. 


\section{L'agent de l'autonomie}

34 nous sommes dans le cadre d'un ensemble d'actions éducatives, il est normal de confondre les acteurs à qui incombe la tâche de réaliser le concept, sans quoi on pourrait légitimement contester son utilité. L'agent sera ce qui détermine la mise en place de l'autonomie. Les séquences répondant à cette préoccupation se situent dans l'environnement du terme, parfois en tête de chapitre. De plus, nous avons retenu les infinitifs qui permettaient de récupérer un agent $(10)^{17}$. Notre travail en a déterminé trois: l'équipe d'adultes (exemples (10 et 11), colonne A5a), les lieux (exemple (12), colonne $\mathrm{A} 5 \mathrm{~b}$ ), enfin les règles régissant la structure ${ }^{18}$ (exemple (13), colonne $\mathrm{A} 5 \mathrm{c}$ ) :

\begin{tabular}{|l|l|}
\hline$(10)$ & Développer l'autonomie. (16-Palaiseau) \\
\hline$(11)$ & $\begin{array}{l}\text { Les équipes d'animation encouragent l'apprentissage de l'autonomie et le sens de la } \\
\text { responsabilité au sein de la collectivité. (18-Meudon) }\end{array}$ \\
\hline$(12)$ & $\begin{array}{l}\text { [les structures d'accueil] doivent concourir au développement et à l'autonomie par } \\
\text { l'aménagement spécifique de l'espace. (23-Antony) }\end{array}$ \\
\hline$(13)$ & $\begin{array}{l}\text { Développer l'autonomie de l'enfant, en élaborant et en contrôlant ensemble des règles de } \\
\text { vie. (26-Cergy) }\end{array}$ \\
\hline
\end{tabular}

On note toutefois qu'un nombre conséquent de projets ne désigne pas d'agent. Ce dernier n'est pas absent formellement, ce qui confirme sa nécessité. Il est mentionné par un ensemble d'expressions qui englobent les agents déterminés plus haut sans en distinguer un seul : projet, le centre de loisirs, les structures.

\section{Les circonstances de l'autonomie}

Avec les circonstances de l'autonomie nous quittons, mais pas encore complètement, le dépouillement des présupposés contenus dans son énonciation. Nous avons relevé ici les circonstances distinctes de l'autonomie qui en conditionnent l'émergence. Sans elles, l'autonomie ne se réalise pas. Ces conditions sont au nombre de quatre dans notre corpus : la sécurité, le groupe, la liberté et la non-consommation.

(a) Plusieurs projets jugent que la sécurité est une condition nécessaire (colonne C1). C'est l'opinion par exemple de (12-Issy) qui lui subordonne les autres actions éducatives :

(14) Assurer la sécurité physique, sanitaire, affective et morale de chaque enfant (conditions indispensables pour aborder d'autres objectifs).

(b) La présence d'un groupe est une condition qui apparaît d'une façon significative (colonne C2). On peut suspecter cette circonstance de véhiculer une idéologie de gauche 
(groupe renvoyant à « collectif »), mais ce soupçon n'est pas confirmé dans le cadre précis auquel nous nous sommes astreint ${ }^{19}$.

(15) Nous pouvons contribuer à donner aux jeunes les moyens de pouvoir être des êtres responsables dans la société de demain, des adultes autonomes, en leur donnant des outils les rendant capables de s'exprimer et d'agir au sein d'une collectivité. (29-Bobigny)

(c) La liberté est liée à l'autonomie dans quelques cas (exemple (16), colonne C3). Ce point mérite deux observations indépendantes. Primo, le fonctionnement hyperonymique liberté/autonomie que Petiot (1991) avait souligné dans le discours du P.S. est corroboré partiellement. Cependant, aucun lien ne peut être établi dans notre corpus avec un étiquetage politique. Secundo, la liaison liberté/autonomie est un point de divergence réel entre les deux repères théoriques éducatifs qui nous accompagnent. Vayer (1993), refusant les acceptions de liberté hors du champ de la psychologie, est conduit à séparer explicitement les deux vocables. Hoosmans-Gosset (1994), elle, insiste à plusieurs reprises sur les liens entre les deux notions. Nous tenons donc dans ce désaccord un point théorique disputé.

(16) Vers l'homme le plus libre ... ce qui suppose l'autonomie et l'épanouissement personnel de chaque enfant. (31-Marly le Roi)

40 (d) La dernière condition n'a pas la portée philosophique des précédentes. Elle est la seule à être formulée d'une manière négative, ce qui en rend la compréhension plus ardue. La consommation est montrée du doigt sans recevoir de contenu. Notons que ce rejet de la consommation est insensible aux clivages politiques. Elle n'est donc pas l'héritière (ou bien ignore l'être) de la « société de consommation »des années 60/70. Autant l'autonomie est fédératrice, autant la consommation est un bouc émissaire universel. De ce fait, l'autonomie et la consommation paraissent antonymes.

(17) Le centre de loisirs doit encourager l'apprentissage de l'autonomie ... cela suppose que l'enfant ... soit acteur de ses loisirs sans pour autant tomber dans une logique de consommation. (6-Massy)

\section{Contenu de l'autonomie}

41 Si l'on s'en tient à ce qui précède, la caractérisation de l'autonomie est pauvre et demeure très abstraite. Certes nous donnons une consistance à la configuration (4), dont l'étude a dégagé plusieurs orientations sémantiques. Certaines sont inconciliables. Une place l'autonomie du côté de l'inné, du droit tandis qu'une autre la voit du côté de l'apprentissage nécessaire, de l'acquis. Toutefois, le corpus ne construit pas encore le sens interne de ce terme. Il reste à expliciter.

Le besoin de définition s'imposait de notre point de vue car il motivait en partie notre question initiale. Mais, c'était oublier qu'on ne définit pas ce qui va de soi, surtout quand 
il ne s'agit pas de vulgarisation. L'autonomie ne soupçonne pas sa fragilité, à l'instar des termes techniques issus d'autres domaines scientifiques (voir Mortureux 1990). C'est bien ce que confirme la majorité des projets de notre corpus. Seuls sept d'entre eux se munissent d'une définition (colonne D0). Pour l'embrayer, on reconnaît des procédés connus (voir Mortureux 1991): parenthèses, deux points explicatifs, énoncés introducteurs - c'est à dire, cela suppose que - titre d'un paragraphe. Plusieurs manifestent clairement le souci d'expliquer, motivés, nous le croyons, par un désir de traduction pratique du concept.

Ces définitions explicites présentent un intérêt patent pour saisir les sèmes constitutifs de l'autonomie. D'autres voies d'accès existent. Certains sèmes sont directement issus des environnements linguistiques (déterminations, épithètes, plan du projet). D’autres sont saisis moins directement. Nous en avons récupéré notamment dans les coordinations, exploitant ainsi une possibilité offerte par Maldidier et Guilhaumou (1984 et 1990). Notre objectif était de répondre à la configuration (18) :

(18) L'autonomie, c'est $\mathrm{X}$

Nous rencontrons des éléments disparates, peu sous-classables. Ils forment un continuum qui irait de l'abstrait au concret ou de la définition théorique à l'illustration pratique. Le corpus, sur ce point surtout, n'a aucune prétention exhaustive. Quelques regroupements sont abusifs. Ils ne se justifient que parce qu'ils sont souvent livrés sous cette forme dans les projets. Par ailleurs, rappelons que nous avons éliminé les éléments que l'on ne retrouvait qu'une seule fois. Ainsi nous avons sorti deux points de définition avancés par le projet (12-Issy les Moulineaux), avoir l'esprit critique et être objectif, parce qu'on ne les repérait à nul autre endroit. Cependant, une exception s'imposait à l'égard d'une singularité rencontrée parce qu'elle justifiait à elle seule la présence du terme dans un des projets (5-Dreux, colonne D10). Les projets permettent donc d'établir la liste suivante pour X : agir et faire seul (exemple (19), colonne D1); faire et assumer des choix (exemple (20), colonne D2); prendre des responsabilités (exemple (21), colonne D3); prendre des initiatives, se prendre en charge (exemple (22), colonne D4); s'exprimer (exemple (23), colonne D5); organiser les activités (exemple (24), colonne D6a); choisir les activités (exemple (25), colonne D6b) ; prendre en charge son hygiène (exemple (26), colonne D7a); savoir manger (exemple (27), colonne D7b) ; respecter le matériel (exemple (28), colonne D8) ; s'informer (exemple (29), colonne D9); travailler pour de l'argent (exemple (30), colonne D10) :

\begin{tabular}{|l|l|}
\hline (19) & $\begin{array}{l}\text { L'autonomie : soit la capacité d'agir seul, de son propre chef et de par sa seule réflexion, } \\
\text { son seul désir. (7-Plaisir) }\end{array}$ \\
\hline$(20)$ & $\begin{array}{l}\text { Favoriser l'acquisition progressive de son autonomie, c'est à dire : rendre capable de faire } \\
\text { des choix, de les assumer [...] (12-Issy) }\end{array}$ \\
\hline$(21)$ & $\begin{array}{l}\text { Nos structures doivent favoriser l'autonomie des enfants, tant sur le plan physique } \\
\text { qu'intellectuel et affectif. Les enfants seront encouragés à prendre des responsabilités [...] } \\
\text { (3-Malakoff) }\end{array}$ \\
\hline
\end{tabular}


(22) Pour [...] favoriser [...] son autonomie, il lui faut une structure où il pourra développer sa capacité à se prendre en charge [...] (14-Gennevilliers)

(23) Développer l'autonomie de l'enfant, [...] en amenant l'enfant à exprimer ses souhaits, ses besoins [...] (26-Cergy)

(24) Le projet doit développer l'esprit d'initiative et l'autonomie par la participation à l'élaboration et à la mise en oeuvre des activités. (4-St Ouen l'Aumône)

(25) Le centre de loisirs doit encourager l'apprentissage de l'autonomie [...] cela suppose que l'enfant ait à choisir entre différentes activités [...] (6-Massy)

(26) La prise en charge par l'enfant de son hygiène corporelle ce qui favorise son autonomie et sa socialisation. (10-Noisy le sec)

(27) Les adultes mangeront avec les enfants et veilleront à la plus grande accession à l'autonomie de tous. (15-Ablis)

(28) Développer l'autonomie de l'enfant : [...] en responsabilisant l'enfant dans le domaine de l'utilisation et du respect du matériel. (26-Cergy)

(29) Nos structures doivent favoriser l'autonomie des enfants, tant sur le plan physique qu'intellectuel et affectif. Les enfants seront encouragés à [...] s'informer [...] (3-Malakoff)

(30) Les contrats emploi-solidarité et les SIVP seront favorisés comme moyen d'acquisition d'une autonomie individuelle. (5-Dreux) 


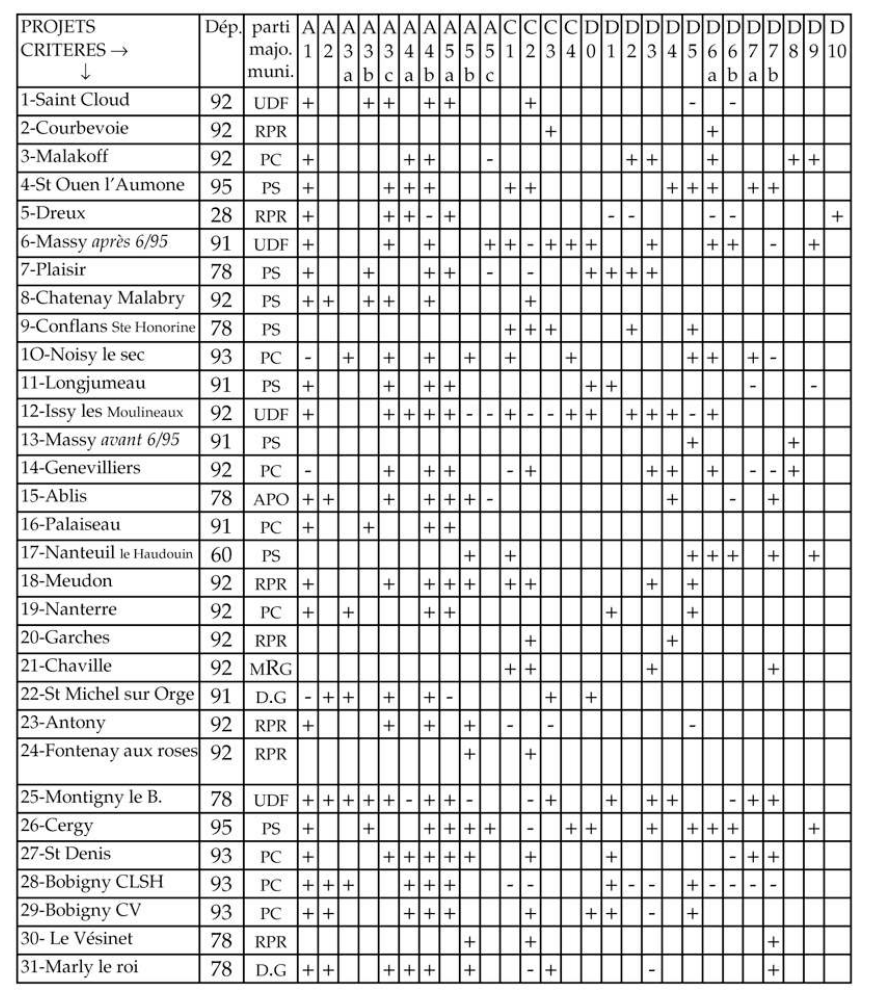

$\mathrm{A} 1$ =présence positive d'auto $\mathrm{A} 2$ =auto est un moyen, par défaut auto est fin en soi $\mathrm{A} 3 \mathrm{a}$ =auto est un droit $\mathrm{A} 3 \mathrm{~b}=$ auto est une qualité à développer $\mathrm{A} 3 \mathrm{c}=$ auto est un acquis $\mathrm{A} 4 \mathrm{a}=$ auto s'applique aux adultes $\mathrm{A} 4 \mathrm{~b}=$ =auto s'applique aux enfants $\mathrm{A} 5 \mathrm{a}=$ =agent est équipe d'adultes $\mathrm{A} 5 \mathrm{~b}=$ =agent est « les lieux » $\mathrm{A} 5 \mathrm{C}=$ agent est « les règles » $\mathrm{C} 1$ = condition de l'autonomie est « la sécurité » $\mathrm{C} 2$ =condition est « le groupe » $\mathrm{C} 3$ =condition est « la liberté » $\mathrm{C} 4$ =condition est « pas de consommation » D1 =agir, faire seul D2 =faire, assumer des choix D3 =prendre des responsabilités D4 =prendre des initiatives, se prendre en charge D5 =s'exprimer D6a =organiser les activités D6b =choisir les activités D7a =vie quotidienne hygiène D7b =vie quotidienne repas D8 =s'informer D9 =respecter le matériel D10 =travailler pour de l'argent

\section{Conclusion : quel vocabulaire de l'éducation?}

Une observation même hâtive du tableau des résultats montre l'extrême hétérogénéité des définitions proposées pour le mot autonomie, quel que soit le champ observé. Les contenus sémantiques ne se superposent pas, sans il est vrai que cela implique qu'ils soient tous contradictoires l'un pour l'autre. Pourtant, on isole une constante: l'autonomie dans l'éducation s'applique à l'individu et non à un collectif d'individus. Elle se démarque ainsi de l'acception usuelle mais également de celle plus débattue issue de l'histoire politique récente, qui contient le sème + collectif quand elle succède à l' autogestion (voir Petiot 1991).

Une interprétation politique du sème + individuel établi pour l'éducation est néanmoins soutenable. L'autonomie serait alors le compromis que la collectivité concède à l'individu, les limites qu'elle assigne à son individualité. On comprend pourquoi l'autonomie ne peut être que positive puisqu'elle est solidaire d'un point de vue : celui du collectif ou de ses représentants, dans notre cas les élus municipaux. D'autres faits corroborent cette proposition. Rappelons tout d'abord la non-pertinence des étiquettes politiques sur 
l'importance du groupe comme condition de l'autonomie. Ce n'est pas le groupe en tant que valeur idéologique qui est revendiqué dans ce cas, c'est le groupe en tant que borne à l'addition des individualités, borne sans laquelle une action politique n'aurait pas de justification (au moins pour les partis de notre corpus). Souvenons-nous également de l'embarras rencontré pour appréhender les relations entre l'autonomie d'une part, la liberté et l'égoïsme d'autre part. L'égoïsme ne diffère de l'autonomie que par l'appréciation subjective de son contenu car l'une et l'autre résultent d'un point de vue du collectif sur l'individu. Cela dit, cette explication ne règle pas tout, mais elle éclaircit partiellement les raisons de l'hétérogénéité des définitions telles qu'elles découlent du tableau. Un compromis est indispensable, mais la teneur ne peut être commune même à notre époque qui prône le consensus.

Certes, on admettra que certaines définitions ne sont pas trop divergentes. Les regrouper serait concevable. Cependant, les classes constituées par ce biais ne répondent pas à des critères exploitables, l'appartenance politique des majorités municipales principalement. Il n'y a pas ou pas encore appropriation de l'autonomie éducative par les partis politiques. De plus, et selon nous, classer les différentes définitions masquerait l'enseignement majeur de l'hétérogénéité constatée. La définition du terme autonomie est intimement liée à son énonciateur, en dépit de la faiblesse des traces qu'il dépose. On comprend alors la force fédératrice du mot. Chacun l'énonce ou le comprend avec la signification qu'il lui a attribuée, sans avoir conscience de la non-universalité de son discours. Nous ne croyons pas exagérer lorsque nous prétendons que l'autonomie se paraphrase par: «ce que je pense que doit être l'homme dans la société quand je l'aurai éduqué ». Cela fonctionne comme la petite fiole dans les tragédies d'Euripide, d'après Aristophane (Les grenouilles).

Faut-il étendre ce constat de la forte relation entre l'énonciateur et la définition d'un terme à l'ensemble du vocabulaire technique de l'éducation? Au moins pour les concepts théoriques, la question est légitime ${ }^{21}$. Pour ce qui est de notre corpus, une approche rapide des expressions répertoriées va dans ce sens sans atteindre toutefois le degré de ce que nous soutenons pour l'autonomie. Cependant, les projets éducatifs, malgré leur richesse, ne constituent qu'une entrée possible dans le vocabulaire des sciences de l'éducation, d'autres accès méritent une investigation.

\section{BIBLIOGRAPHIE}

BEACCO, J.-Cl. et MOIRAND S. (1995) Autour des discours de transmission des connaissances, Langages $\mathrm{n}^{\circ} 117$

BERGE, A. (1955) La liberté dans l'éducation, Paris, Scarabée

BÖHM, W. (1994) Maria Montessori, in Quinze pédagogues Leur influence aujourd'hui J. Houssaye ed., Paris :Armand Colin

BONNAFOUS, S. (1991) Quand la presse catholique parle de laïcité, Mots 27

BONNAFOUS S. et TOURNIER M. (1995) Analyse du discours, lexicométrie, communication et politique, Langages $\mathrm{n}^{\circ} 117$ 
ELBAZ S. (1994) Prélude au compromis, Kimé

HOFFMANS-GOSSET, M.-A. (1994) Apprendre l'autonomie Apprendre la socialisation, Lyon :Chronique Sociale

MAINGUENEAU, D. (1995) Présentation, Langages nº 117

MALDIDIER, D. et GUILHAUMOU, J. (1984) Coordination et discours. « Du pain et X » à l'époque de la révolution française, $\operatorname{LINX} \mathrm{n}^{\circ} 10$

MALDIDIER, Denise, GUILHAUMOU, Jacques, (1990), « De nouveaux gestes de lecture ou le point de vue de l'analyse de discours sur le sens », in La quadrature du sens, Claudine Normand éd. Paris, PUF

MILNER, J.-Cl. (1984) De l'école, Paris :Seuil

MORTUREUX, M.-F. (1990) Fonctionnement des termes scientifiques et lexique, in La quadrature du sens, Claudine Normand ed. Paris, PUF

MORTUREUX, M.-F. (1991) Néologie lexicale et énonciation personnelle dans le discours scientifique, in LINX numéro spécial : Etudes de Linguistique française à la mémoire d'Alain Lerond, Michèle Perret et André Eskénazi eds.

PETIOT, G. (1990) «Liberté(s) » dans les discours politiques, in La quadrature du sens, Claudine Normand ed. Paris, PUF

PETIOT, G. (1991) D’autogestion à autonomie, où en est le socialisme démocratique ? Mots n 22

PETIOT, G. (1995) Voile, Tchador ou foulard ? Problèmes de dénomination dans les discours des médias, Les carnets du CEDISCOR n ${ }^{\circ} 3$

VAYER, P. (1993) Le principe d'éducation et l'autonomie. Paris, ESF Editeur

\section{NOTES}

1. Ces deux travaux ne sont pas dupes de l'imprécision sémantique de l'autonomie. Ils s'appliquent l'un et l'autre à lui donner une consistance que nous n'avons pas tenue comme modèle. Cependant, de par leur statut scientifique, ces ouvrages accompagneront notre étude.

2. C'est en tout cas l'ambiance du moment : les exemples étrangers et expérimentaux (Epinal entre autres) foisonnent actuellement sur le petit écran. Les propositions récentes de Guy Drut sur l'aménagement des rythmes scolaires ont d'ailleurs enthousiasmé les partisans de ce « rapprochement » (voir notamment le pré-rapport de la commission Fauroux).

3. Ce projet évite ainsi une implication du texte ministériel. Celui-ci contient une obligation qui ne va pas de soi parce qu'elle-même idéologique : les organisateurs (ici les communes) doivent avoir des conceptions éducatives. Nous rapprochons cette curiosité de la critique virulente de Milner (1984) à propos du lien inévitable entre l'école et le " pédagogique » : « toute personne qui parle de l'école tient pour obligée la référence pédagogique » (p. 71).

4. Par exemple le critère " groupe » (voir infra) défini dans la socialisation.

5. L'absence de contraintes de contenu oppose ainsi Jeunesse et Sports et Education Nationale. Il n'y a pas de programme officiel pour les loisirs des jeunes. Remarquons que le ministère, dans l'ensemble des textes réglementaires n'utilise jamais le terme autonomie. On le trouve tout de même dans les commentaires d'un arrêté concernant les adolescents (lettre $n^{\circ} 721$ du 31/7/81). 6. Rappelons que celui-ci est peu néologique. On notera le mot repère (8/31), qui est souvent dans des environnements linguistiques similaires à autonomie : «L'enfant a besoin d'autonomie....de 
repères ». Il n'est jamais défini, ce qui, pour ne pas y voir un paradoxe, oblige à le considérer comme un terme technique.

7. L'éducation ne se comporte pas ainsi avec tout son vocabulaire technique. Le terme laïcité est également revendiqué de tous. Bonnafous (1991) montre que la presse catholique se l'approprie également avec des contenus délibérément différents.

8. Pour ces aspects historiques, voir Petiot (1991).

9. Nous comparons partiellement cette distanciation avec celle qu'on entend quelquefois avec le terme collaboration qui a gardé pour certains son histoire.

10. A plusieurs reprises une autre fonction apparaît : l'autonomie est un besoin. Cette fonction est compatible avec les deux que nous avons retenues.

11. C'est le cas de la lettre du ministère citée à la note $5:$ «Les nouvelles orientations visent essentiellement à....promouvoir une plus grande autonomie des jeunes ».

12. Une petite réserve : deux projets ajoutent les adjectifs personnelle et individuelle sans pour autant ouvrir la possibilité d'une autonomie collective.

13. La comparaison entre Böhm (1994) et Berge (1955) est révélatrice. Dans Böhm (1994) on trouve une analyse critique de la pédagogue Maria Montessori. Sur les 18 pages qui lui sont consacrées on trouve le mot autonomie à huit reprises dans les cinq dernières pages, celles qui décrivent l'aspect concret de cette pédagogie. Dans Bergé (1955), l'auteur consacre un long passage aux thèses de la pédagogue italienne (p. 82-94) principalement d'un point de vue pratique. Pas une seule occurrence d'autonomie, bien que l'auteur utilise le terme dans d'autres circonstances.

14. A une exception près : 5-Dreux. Un projet (22-St Michel) n'attribue l'autonomie qu'aux jeunes enfants.

15. En revanche (i) est le sens qui est mis en avant dans Vayer (1993). Pour l'auteur, l'enfant «exerce» son autonomie car l'enfant «est parfaitement autonome», sauf quand l'autonomie est «bloquée» ou «perturbée» ou «déformée». Une cause importante de ce blocage est l'attitude de l'adulte.

16. Avec toutes les conséquences de cette image : les variations peuvent apparaître sur le début du parcours ou sa fin. Notons par exemple la quête de l'autonomie, que nous interprétons comme un but inaccessible.

17. Notons que l'agent est dans ce cas toujours un humain pour des raisons syntacticosémantiques.

18. Deux autres agents sont avancés à une seule reprise : les enfants et les activités adaptées.

19. Toutefois, l'importance du groupe est clairement plus affirmée dans les projets éducatifs des municipalités communistes que dans les communes de l'actuelle majorité.

20. Rappelons qu'un critère d'observation sera visible du point de vue de la réalisation (présence du signe + ou -) et de sa participation à la définition de l'autonomie (signe + si l'autonomie est + ou - colonne A1).

21. Une preuve extralinguistique appuie la liaison intime ressentie entre l'énonciateur et son discours éducatif. A l'exception d'Issy les Moulineaux, de Meudon et surtout des villes communistes (Bobigny, StDenis, Nanterre), nous nous sommes procuré les projets éducatifs difficilement, après plusieurs démarches. Très souvent, on nous a opposé un refus sous le prétexte faux qu'il s'agissait d'un texte « privé ». 


\section{RÉSUMÉS}

Le mot autonomie est un terme technique du domaine éducatif. Pour définir ce terme, on s'est appuyé sur un corpus constitué de projets éducatifs de municipalités. Ce texte a la singularité d'être le seul texte qui oblige les élus à formuler leurs conceptions éducatives. Il s'adresse à des praticiens, ce qui justifie le peu de définitions explicites de l'autonomie dans le corpus. L'étude des présupposés à l'emploi du terme ainsi que le relevé des éléments explicatifs fait émerger deux invariants :

(a) le mot autonomie est non seulement positif mais encore fédérateur. Les projets sont d'accord sur le mot, ignorant qu'ils peuvent ne pas l'être sur le contenu.

(b) au regard du sens politique issu de l'histoire récente ou tout simplement du sens usuel, l'autonomie ne retient que le seul sème +individuel. Hormis cette constante, les définitions extraites par l'outillage linguistique sont extrêmement variées, souvent inconciliables.

L'étude proposée trouve alors sa structuration dans deux hypothèses complémentaires :

(i) l'autonomie est le point de vue du collectif sur l'individu, on comprend du coup pourquoi le terme est fédérateur.

(ii) le contenu du compromis obligé est forcément variable. A défaut d'une prise de position des partis politiques sur l'autonomie éducative, l'appropriation quasi complète du terme par l'énonciateur est de rigueur.

In the educational field, the word autonomie is a technical one. In order to give this term a definition, a corpus of educational projects coming from city councils has been used. These documents are unusual in that they force the council's members to formulate their own educational conception. They apply to practitioners, which justifies the few explicit definitions found in the corpus. The survey of presuppositions contained in the use of autonomie reveals two regularities : on the one hand, the word autonomie is not only positive, but also federative. Thus, various projects agree on the form of autonomie, but know nothing whatever about they might diverge on its meaning. On the other hand, through either its political or its ordinary meaning, autonomie features the [+ individual] sememe. Among these regularities, definitions remain extremely diversified, and even incompatible with each other. This survey has recourse to the following complementary hypothesis : first, the notion of "autonomy" is the point of view of the collective aspect on the individual one. In the second place, the content of the necessary compromise is inevitably variable. For lack of strong line taken by political parties about the notion of "educational autonomy", the total appropriation of the referring word by the speaker seems to be compulsory.

\section{AUTEUR}

\section{MUSTAPHA KRAZEM}

